\title{
ON SOME INEQUALITIES EQUIVALENT TO THE WRIGHT-CONVEXITY
}

\section{ANDRZEJ OLBRYŚ}

Abstract. In the present paper we establish some conditions and inequalities equivalent to the Wright-convexity.

Mathematics subject classification (2010): 39B62, 26A51, 26B25.

Keywords and phrases: Convexity, Jensen-convexity, Wright-convexity.

\section{REFERENCES}

[1] B. C. Arnold, A. W. Marshall, I. Olkin, Inequalities: Theory of Majorization and Its Applications, Springer Series in Statistics, New York-Dordrecht-Heidelberg-London, 2011 (Second Edition).

[2] V. Ciobotariu-Boer, Hermite-Hadamard and Fejér Inequalities for Wright-convex functions, Octogon Math. Magazine 17, 1 (2009), 53-69.

[3] S. S. DRAGOMIR, Two mappings in connection to Hadamard's inequalities, Math. Anal. Appl. 167, 1 (1992), 49-56.

[4] S. S. Dragomir And C. E. M Pearce, Selected Topics on Hermite-Hadamard Inequalities and Applications, RGMIA Monographs, Victoria University, 2002.

[5] N. Elezović, J. PeČArić, A note on Schur-convex functions, Rocky Mountain J. Math. 30, 3 (2000).

[6] J. HADAMARD, Étude sur les properiétés entiéres et en particulier dúne fonction considerée par Riemann, J. Math. Pures Appl. 58 (1893), 171-215.

[7] G. Hardy, J. E. LitTlewood And G. Pólya, Inequalities, 1st ed., 2nd ed., Cambridge Univ. Press, Cambridge, London and New York, 1934, 1952.

[8] Ch. Hermite, Sur deux limites dúne intégrale définie, Mathesis 3 (1883), 82.

[9] M. In Ho, Fejér inequalities for Wright-convex functions, J. Inequal. Pure and Appl. Math. 8, 1 (2007), Art. 9, 9 pp.

[10] Z. Kominek, Convex Functions in Linear Spaces, Prace Naukowe Uniwersytetu Ślạskiego w Katowicach nr. 1087, Katowice, 1989.

[11] Z. KomineK, On additive and convex functionals, Radovi Mat. 3 (1987), 267-279.

[12] M. KUCZMA, An introduction to the theory of functional equations and inequalities, Polish Scientific Publishers and Silesian University Press, Warszawa-Kraków-Katowice, 1985.

[13] Gy. Maksa, Zs. PÃLeS, Decomposition of higher-order Wright-convex functions, J. Math. Anal. Appl. 359 (2009), 439-443.

[14] C. T. NG, Functions generating Schur-convex sums, W. Walter (Ed.), General Inequalities, S. Oberwolach, 1986, Internat Ser. Numer. Math. vol. 80, Birkhäuser, Boston, 1987, pp. 433-438.

[15] C. P. Niculescu And L. E. Persson, Convex Functions and their Applications, A Contemporary Approach CMS Books in Mathematics vol. 23, Springer-Verlag, New York, 2006.

[16] K. Nikodem, On some class of midconvex functions, Ann. Polon. Math. 50 (1989), 145-151.

[17] A. OlBRYś, Representation theorems for t-Wright convexity, J. Math. Anal. Appl. 384, 2 (2011), 273-283.

[18] J. PeČarić, F. Proschan, Y. L. Tong, Convex Functions, Partial Orderings, and Statistical Applications, Acad. Press, Inc., Boston, 1992.

[19] A. W. Roberts, D. E. Varberg, Convex Functions, Academic Press, New York and London, 1973.

[20] R. T. Rockafeller, Convex Analysis, Princeton Univ. Press, Princeton, NJ, 1970.

[21] I. SCHUR, Über eine Klasse von Mittelbidungen mit Anwendungen auf die Determinanten theorie, Sitzunsber. Berlin. Math. Ges. 22 (1923), 9-20. 
[22] Brian S. Thomson, Symmetric properties of real functions, Pure and Applied Mathematics, New York-Basel-Hong Kong, 1994.

[23] K. L. TsEng, G. S. YANG AND S. S. DRAGomiR, Hadamard inequalities for Wright-convex functions, Demonstratio Math. 37, 3 (2004), 525-532.

[24] E. M. Wright, An inequality for convex functions, Amer. Math. Monthly 61 (1954), 620-622.

[25] L. Zalinescu, Convex Analysis in General Vector Spaces, World Scientific, New Jersey, 2002. 